\title{
Generic ordering of structural transitions in quasi-one-dimensional Wigner crystals
}

\author{
J. E. Galván-Moya, ${ }^{*}$ V. R. Misko, ${ }^{\dagger}$ and F. M. Peeters ${ }^{\ddagger}$ \\ Department of Physics, University of Antwerp, Groenenborgerlaan 171, B-2020, Antwerp, Belgium \\ (Received 10 June 2014; revised manuscript received 28 August 2014; published 17 September 2014)
}

\begin{abstract}
We investigate the dependence of the structural phase transitions in an infinite quasi-one-dimensional system of repulsively interacting particles on the profile of the confining channel. Three different functional expressions for the confinement potential related to real experimental systems are used that can be tuned continuously from a parabolic to a hard-wall potential in order to find a thorough understanding of the ordering of the chainlike structure transitions. We resolve the long-standing issue why the most theories predicted a 1-2-4-3-4 sequence of chain configurations with increasing density, while some experiments found the 1-2-3-4 sequence.
\end{abstract}

DOI: 10.1103/PhysRevB.90.094111

PACS number(s): 81.30.-t, 37.10.Ty, 82.70.Dd, 52.27.Lw

\section{INTRODUCTION}

A crystalline structure consists of a periodic arrangement of molecules, atoms, or different particles. The first prediction about self-arrangement of particles, nowadays known as Wigner crystals (WCs) [1], states that in the absence of kinetic energy, a system of interacting particles arranges itself into a body-centered cubic (bcc) lattice in three-dimensional (3D) space [2,3], a triangular lattice in two dimensions (2D) [4-6], while in one dimension (1D), the energetically most favorable organization is given by an evenly spaced lattice [6-8].

For a quasi-one-dimensional (Q1D) system, Piacente et al. [9,10] studied the ground state (GS) configuration of a system of particles confined in a parabolic channel, and found a nonsequential ordering of transitions (non-SOT) between 1-2-4-3-4-5-6-chainlike structures with increasing particle density. They revealed that this ordering of transitions between chains is robust, being not affected by the range of the interaction between the particles [11-13]. That succession of phases differs from a sequential ordering of transitions (SOT), which is characterized by a consecutive succession of phases with 1-2-3-4-5-6 chains, as one would intuitively expect to be the case. The structural transition from a two- to a four-chain configuration occurs, in the case of a non-SOT, through a zigzag transition of each of the two chains and a simultaneous small shift along the chain, which makes it a discontinuous transition [10].

The only second-order transition in this sequence is the zigzag transition between the one- and the two-chain configuration, which has been extensively studied in classical [12-16] and quantum [6,17-21] systems. A detailed analysis of the structural transitions for larger number of chains has to a lesser extent also been addressed [9,10,22,23]. Experimental findings in a colloidal Q1D system showed evidence of transitions from eight- up to five-chain configurations [22]. Numerical calculations in the same work, suggested that this sequence continues reducing the number of chains, one by one, until the three-chain configuration. No information was provided about the transition between two- and three-chain structures.

However, a direct transition from the two- to the threechain configuration, 2-3, has been shown to take place in a

\footnotetext{
*JesusEduardo.GalvanMoya@uantwerpen.be

†vyacheslav.misko@uantwerpen.be

†Francois.Peeters@uantwerpen.be
}

number of systems. For example, for Yukawa particles the direct 2-3 transition was observed in dusty plasma clusters [24] with increasing linear density. In addition, a SOT has been predicted theoretically for an Abrikosov-vortex arrangement in a superconducting slab for low temperatures [25-29], for Pearl vortices [30], and also for binary mixtures of repulsive particles [31], in particular, when the ratio between charges of both species was around $1 / 5$.

These examples suggest that, in spite of the demonstrated robustness of the non-SOT with respect to the range of the interaction between the particles, the nonsequential ordering is perhaps sensitive to the system parameters and conditions. First, the real confinement can be different from parabolic. For example, in the case of colloids [22] the boundaries could be closer to hard walls. In the case of superconducting vortices, the potential barrier preventing vortices from entering or escaping the slab is described by the known Bean-Livingston barrier [32,33], which for a wide slab is very different from a parabola. In addition, fluctuations of any nature can be responsible for the disappearance of the non-SOT. This was probably the case in the experiment [34] that analyzed the melting of the WC chainlike structures and their transport in a Q1D channel of electrons on a liquid He surface. In particular, the non-SOT has been observed in that experiment for very low temperatures, while even at $T=1 \mathrm{~K}$ the non-SOT regime was washed out, and the usual SOT was observed instead. This behavior is in agreement with the early predictions by Piacente et al. [10] showing that thermal fluctuations can easily destroy the non-SOT. This finding is also in agreement with recent computer simulations [35] on the dynamics of WCs in Q1D channels with constrictions. It was shown that even in the absence of thermal fluctuations, the non-SOT observed in long constrictions was destroyed in short constrictions, due to fluctuations of the number of particles flowing through the constriction.

Although fluctuations are generally a universal "tool" to destroy any ordering, and as shown in the examples above also the non-SOT, the role of other factors such as the functional form of the confinement potential remains unexplored. In particular, an important open question is: How universal is the non-SOT? Is it typical for systems with parabolic confinement, or is it of a more generic nature? The positive answer to the latter would open broader possibilities for experimental observation of the non-SOT, provided the fluctuations are very weak. This motivated us to investigate the universality of the non-SOT. 
In the present work we study the influence of the confinement potential on the GS of a Q1D system of interacting particles, elucidating the general model of the order of the transitions between chainlike structures. Different confinement potentials are used in order to study the behavior of the GS transitions, when the profile of the channel is varied continuously from a paraboliclike to a hard-wall potential.

\section{MODEL SYSTEM}

We consider an infinite system of identical interacting particles with mass $m$ and charge $q$, which are trapped in a Q1D channel through an external confinement potential, restricting the movement of the particles in the $y$ direction. The total energy of the system is given by the following expression:

$$
H=\sum_{i=1}^{\infty} \sum_{j>i}^{\infty} V_{\text {int }}\left(\left|\mathbf{r}_{i j}\right|\right)+\sum_{i=1}^{\infty} V_{\text {conf }}\left(y_{i}\right),
$$

where $\mathbf{r}_{i j}=\mathbf{r}_{i}-\mathbf{r}_{j}$ is the relative position of the $i$ th with respect to the $j$ th particle in the system, while $V_{\text {int }}(r)$ and $V_{\text {conf }}(y)$ represent the pairwise interparticle interaction and the confinement potential of the channel, respectively.

The interparticle interaction, in dimensionless units, is taken as follows:

$$
V_{\text {int }}(r)=\frac{e^{-\kappa r}}{r},
$$

where the parameter $\kappa$ allows us to tune the range of the interaction between particles in the system. In order to understand the effect of confinement on the ordering of the phase transitions, we considered the following three different functional forms for the confinement potential:

$$
\begin{aligned}
& V_{A}(\alpha, y)=v^{2} y_{0}^{2-\alpha}|y|^{\alpha}, \\
& V_{B}(\beta, y)=v^{2} y_{0}^{2} \frac{\cosh (\beta y)-1}{\cosh \left(\beta y_{0}\right)-1}, \\
& V_{C}(\gamma, y)=\sigma^{2} y_{0}^{2}\left[e^{-\gamma^{2}\left(y-y_{0}\right)^{2}}+e^{-\gamma^{2}\left(y+y_{0}\right)^{2}}\right],
\end{aligned}
$$

where $y_{0}$ determines the effective width of the confinement channel, while the parameters $\alpha, \beta$, and $\gamma$ allow one to control the sharpness of its profile and the dimensionless frequencies $v$ and $\sigma$ control the strength of the confining potential.

In order to put these potentials in the context of possible experimental, the energy is expressed in units of $E_{0}=$ $\left(m \omega_{0}^{2} / 2\right)^{1 / 3}\left(q^{2} / \epsilon\right)^{2 / 3}$ and all distances are expressed in units of $r_{0}=\left(2 q^{2} / m \omega_{0}^{2} \epsilon\right)^{1 / 3}$, where $\omega_{0}$ measures the strength of the confinement potential and $\epsilon$ is the dielectric constant of the medium the particles are moving in. Those definitions allow one to generalize the pairwise interaction as $V_{\text {int }}(r)=$ $q^{2} \exp (-\kappa r) / \epsilon r$. The dimensionless linear density $\eta$ is defined as the number of particles per unit of length along the unconfined direction.

Previously, it has been shown that, in a parabolic channel, the sequence of transitions in the GS configuration of the system when increasing the density is not affected by the range of the interaction between particles, when the pairwise potential is modeled by a screened Coulomb potential [10]. This pairwise potential has been found as a good candidate to model the interparticle interaction in different experimental systems in condensed matter physics as colloidal systems [36], charged particles in a dusty plasma [37], system of microscopic charged particles [38-41], and even in systems of vortices in superconductors [42]. In the last two cases the pairwise interaction considered is the modified Bessel function $K(x)$, which behaves very similarly to the potential we propose in the present study.

In the present work the range of the interaction is fixed by choosing $\kappa=1$, excluding the long-range Coulomb interaction $(\kappa=0)$ from our study. In order to analyze the influence of the profile of the channel on the sequence of
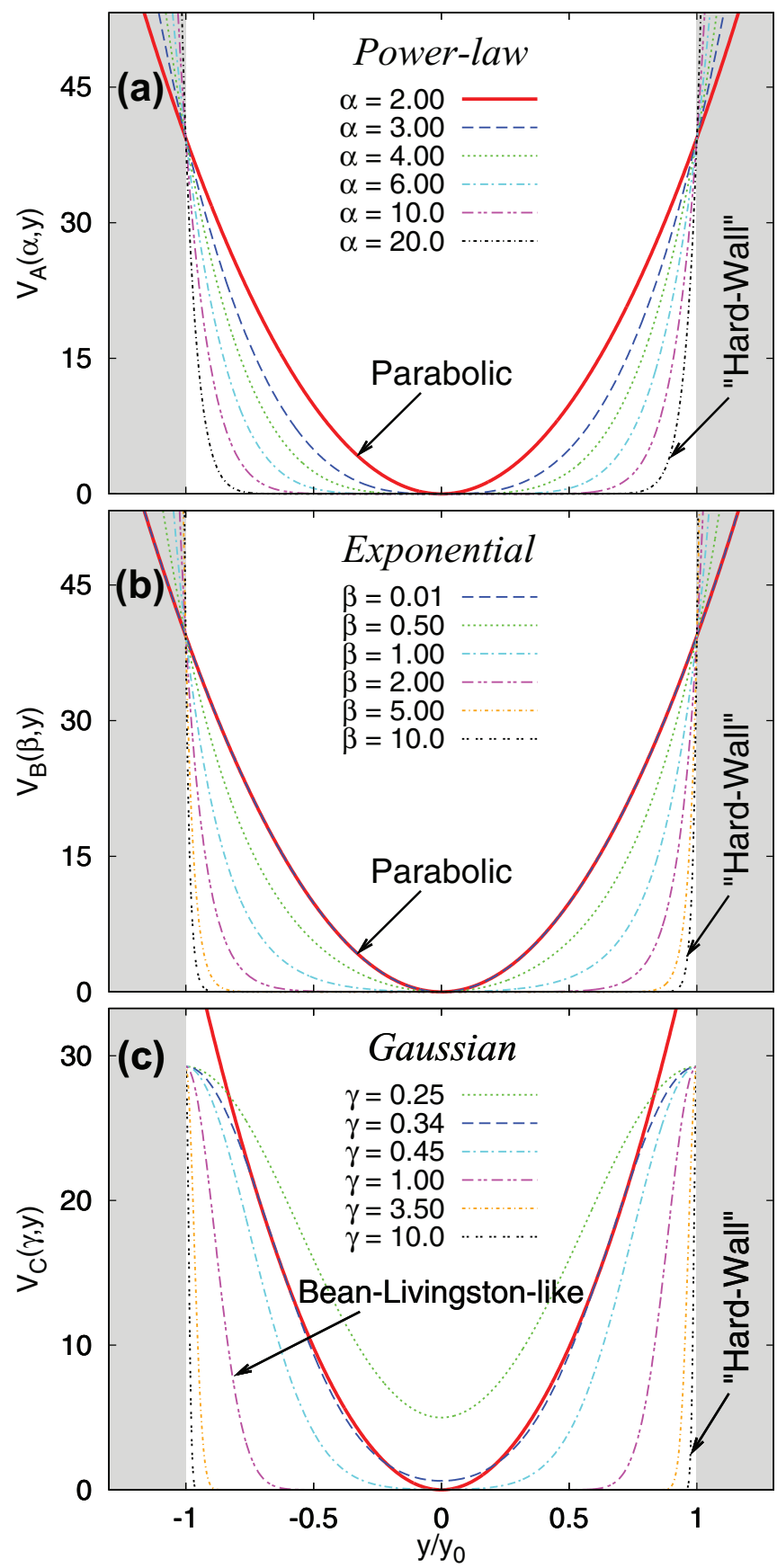

FIG. 1. (Color online) Profile of the confinement potentials considered in this work, for different values of the shape parameters: (a) power-law, (b) exponential-like, (c) Gaussian (see details in the text). The solid red curve shows the parabolic potential for reference, and the hard-wall potential is indicated by the white region in the middle of the figure. 
the phase transitions, we fix the confinement strength for the parabolic potential [i.e., $\alpha=2$ in Eq. (3)] to $v=1$. Next we note that $V_{B}(\beta=0, y)=V_{A}(\alpha=2, y)$ is a parabola. For $V_{C}(\gamma, y)$ we determine the parameters $\sigma$ and $y_{0}$ such that for some $\gamma$ value we obtain a confinement potential that is very close to a parabola [see Fig. 1(c)]. The fitting results in the choice of parameters $\sigma=0.862$ and $y_{0}=6.275$, which are fixed for all numerical calculations performed in the present work. The different confinement potentials are plotted in Fig. 1 for different values of the shape parameters.

Figure 1 shows the flexibility of the confinement potentials defined by Eqs. (3)-(5). Thus, by gradually changing the shape parameters $\alpha, \beta$, and $\gamma$, we follow a continuous evolution from a soft paraboliclike [note that in Fig. 1(c), due to the shape of the confinement potential given by Eq. (5), the closest approximation to the parabolic confinement is found for $\gamma=$ 0.34 ; this profile is being plotted with a blue dashed line] to the hard-wall potential. As a reference, the parabolic profile is shown by the red curve in each plot. It is worth noting that the three functional forms for the confinement potential are essentially different and approach the hard-wall limit in a different manner. The potential profiles were chosen such that they model confinement potentials in various physical systems ranging from charged particles and colloids in narrow channels to vortices in superconducting stripes. For example, the potential profile in Fig. 1(c) for $\gamma=1$ models the BeanLivingston barrier for vortex exit from a superconductor.

\section{TRANSITIONS BETWEEN CHAINLIKE STRUCTURES}

The GS of the system of interacting particles in a Q1D channel consists of chainlike structures [12-15], and the transitions between them are of first order [10,22,23], with the exception of the zigzag transition between one and two chains which is of second order $[12,13]$. Some typical chainlike configurations are shown in Fig. 2, where the order parameters are indicated in red.
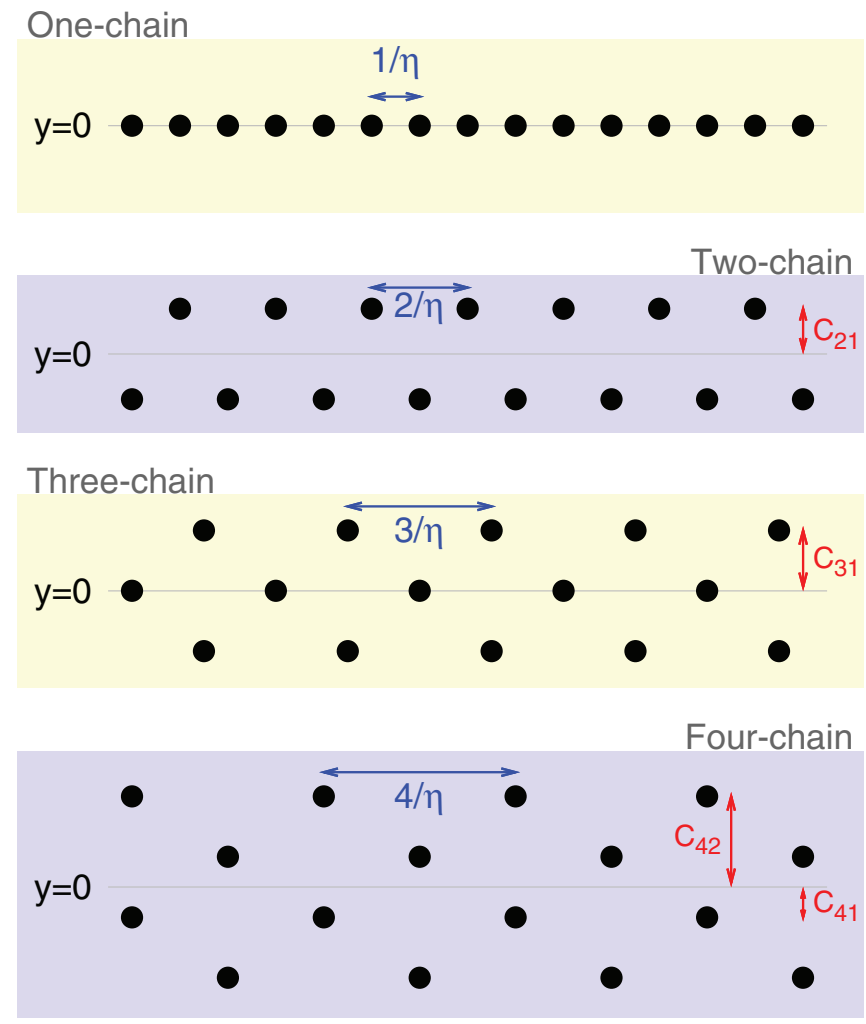

FIG. 2. (Color online) Schematic view of the chainlike configurations together with the relevant order parameters used in the calculations, where $\eta$ represents the linear particle density in the unconfined direction.

In order to study the structural transitions in an infinite system of particles interacting through the potential $V_{\text {int }}(r)$ and confined in a Q1D channel defined by $V_{\text {conf }}(y)$, we calculate the total energy of the system for some typical $n$-chain structures, as follows:

$$
\begin{aligned}
H_{1 c h}= & V_{\text {conf }}(0)+\sum_{m=1}^{\infty} V_{\text {int }}\left(\frac{m}{\eta}\right) \\
H_{2 c h}= & V_{\text {conf }}\left(\frac{c_{21}}{\eta}\right)+\sum_{m=1}^{\infty} V_{\text {int }}\left(\frac{2 m}{\eta}\right)+\sum_{m=1}^{\infty} V_{\text {int }}\left[\frac{2}{\eta} \sqrt{c_{21}^{2}+\left(m-\frac{1}{2}\right)^{2}}\right], \\
H_{3 c h}= & \frac{1}{3} V_{\text {conf }}(0)+\frac{2}{3} V_{\text {conf }}\left(\frac{3 c_{31}}{\eta}\right)+\frac{1}{3} V_{\text {int }}\left(\frac{6 c_{31}}{\eta}\right)+\sum_{m=1}^{\infty} V_{\text {int }}\left(\frac{3 m}{\eta}\right)+\frac{2}{3} \sum_{m=1}^{\infty} V_{\text {int }}\left(\frac{3}{\eta} \sqrt{4 c_{31}^{2}+m^{2}}\right) \\
& +\frac{4}{3} \sum_{m=1}^{\infty} V_{\text {int }}\left[\frac{3}{\eta} \sqrt{c_{31}^{2}+\left(m-\frac{1}{2}\right)^{2}}\right], \\
H_{4 c h}= & \frac{1}{2} V_{\text {conf }}\left(\frac{4 c_{41}}{\eta}\right)+\frac{1}{2} V_{\text {conf }}\left(\frac{4 c_{42}}{\eta}\right)^{2}+\frac{1}{2} V_{\text {int }}\left(\frac{4\left(c_{41}+c_{42}\right)}{\eta}\right)+\sum_{m=1}^{\infty} V_{\text {int }}\left(\frac{4 m}{\eta}\right) \\
& +\sum_{m=1}^{\infty} V_{\text {int }}\left(\frac{4}{\eta} \sqrt{\left(c_{41}+c_{42}\right)^{2}+m^{2}}\right)+\frac{1}{2} \sum_{m=1}^{\infty} V_{\text {int }}\left[\frac{4}{\eta} \sqrt{4 c_{41}^{2}+\left(m-\frac{1}{2}\right)^{2}}\right] \\
& \left.+\frac{1}{2} \sum_{m=1}^{\infty} V_{\text {int }}\left[\frac{4}{\eta} \sqrt{4 c_{42}^{2}+\left(m-\frac{1}{2}\right)^{2}}\right]+\sum_{m=1}^{\infty} V_{\text {int }}\left[\frac{4}{\eta} \sqrt{\left(c_{42}-c_{41}\right)^{2}+\left(m-\frac{1}{2}\right.}\right)^{2}\right],
\end{aligned}
$$




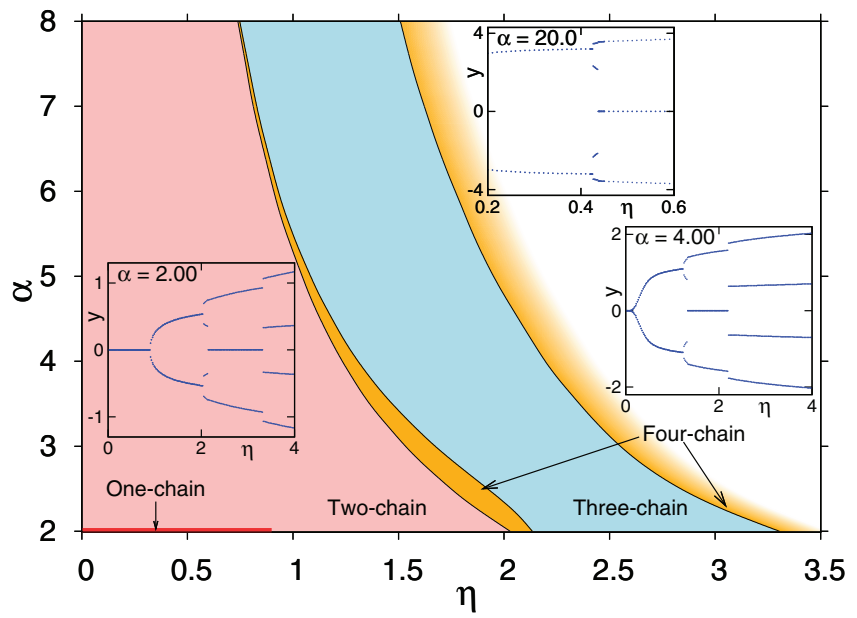

FIG. 3. (Color online) Phase diagram of the ground state for a system with confinement $V_{A}(\alpha, y)$, as a function of $\alpha$ and the linear density $\eta$. All phase transitions are of first order except the zigzag transition which is only possible for $\alpha=2$. The insets show the $y$ position of the particles as a function of $\eta$ for different values of $\alpha$ as indicated in each figure.

where all the distances are expressed in units of the distance between adjacent particles in each chain, as indicated in Fig. 2.

We find the GS of the system by minimizing the energy (numerically) with respect to the order parameter(s) for each chainlike structure.

\section{A. Power-law confinement}

In the case of power-law confinement [Eq. (3)], the phase diagram of the GS is shown in Fig. 3 as a function of the shape parameter $\alpha$ and the linear density $\eta$. As was analytically demonstrated in Refs. [12,13], the stability of the one-chain configuration as the GS is only guaranteed for the case of $\alpha=2$, while for larger values of $\alpha$ the one-chain configuration is no longer found as the GS. This result is represented in Fig. 3 by the thick red line (for $\alpha=2$ ) showing the small region $(0<\eta<0.9)$ where the one-chain structure is found as the GS. The $y$ position of the particles forming the GS, for $\alpha=2$, is presented as a function of $\eta$ in the left-hand side inset of Fig. 3, where the non-SOT is clearly present (i.e., the transitions 2-4-3).

For $\alpha>2$ and small $\eta$, the two-chain configuration is the GS of the system even at low densities, where the interchain distance (i.e., the order parameter $c_{21}$ ) slowly decreases but never becomes exactly zero [13] except for $\eta \rightarrow 0$. This behavior is illustrated in the right-hand side inset in Fig. 3 for $\alpha=4$, where one can also see that a direct transition between the two- and the three-chain configuration is not found (i.e., non-SOT). Indeed, a small region where the four-chain arrangement is the GS remains between the two- and the three-chain structures, even for large values of the shape parameter $\alpha$. Thus the GS transition between two-, four-, and three-chain configurations is still present for $\alpha=20$ (i.e., close to the hard-wall limit), as shown in the upper inset in Fig. 3.

With further increasing the density, the three-chain GS configuration is found as the ordered sequence of the GS configurations with the number of chains increasing one by one (the transitions between the states are of first order), i.e., three-, four-, five-, six-chain, etc.

Therefore, a system of particles confined by a power-law potential [Eq. (3)], shows a robust nonsequential transition between two-, four-, and three-chain configurations as the ground states, for a broad range of densities $\eta$ and the shape of the confinement profile varying from parabolic to hard wall.

\section{B. Exponential type of confinement}

The parabolic potential is the simplest and often used form to model quasi-one-dimensional systems of, e.g., charged particles [9,10], colloids [22], and dusty plasma [24,43]. Another useful form of the confinement potential is the one with exponentially decaying barriers, as described by Eq. (4) that uses a hyperbolic cosine, where $\beta$ acts as a parameter which controls the shape of the channel. The advantage of this form of confinement potential is that, by tuning the control parameter $\beta$, it rapidly evolves into a flat central part in the potential profile providing a fast continuous transition to a hard-wall-like profile [see Fig. 1(b)].

The phase diagram for the GS of a system of particles confined by the potential $V_{B}(\beta, y)$ is shown in Fig. 4 as a function of the parameters $\beta$ and $\eta$.

The limiting case of a parabolic potential is recovered by setting $\beta=0$. The $y$ coordinate of the particles as a function of $\eta$ is shown in the right-hand side inset of Fig. 4 for $\beta=0.01$. With increasing $\beta$, the transitions between chainlike configurations occur at lower values of the density, and the transition between one- and two-chain configurations (zigzag transition) is of second order (indicated by the dashed curve in Fig. 4). Opposite to the above case of a parabolic confinement, the zigzag transition is always stable, even for large values of $\beta$.

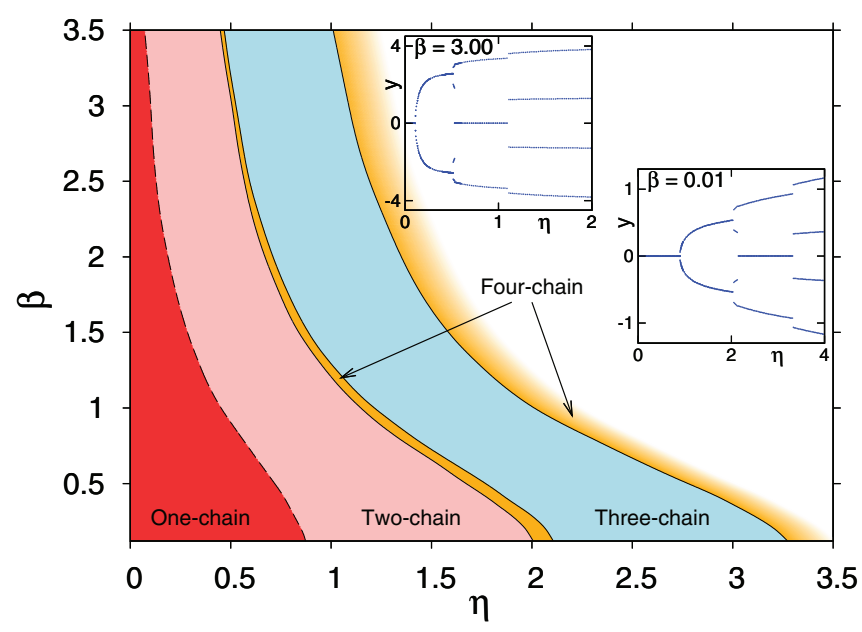

FIG. 4. (Color online) Phase diagram of the ground state for a system with confinement $V_{B}(\beta, y)$, as a function of $\beta$ and the linear density $\eta$. The solid and dashed lines represent first- and second-order transitions, respectively. The insets show the $y$ position of the particles as a function of $\eta$ for different values of $\beta$ as indicated in each figure. 
Therefore, the evolution of the GS of the system is guided by a non-SOT irrespective of the value of $\beta$, thus allowing the emergence of the four-chain state between the two- and threechain configurations. Although the width of this intermediate four-chain region slowly decreases with increasing $\beta$, it does not disappear when the channel profile approaches the hardwall potential, as shown for $\beta=3$ at the upper inset in Fig. 4. It is worth noting that, although this profile evolution (i.e., from a parabolic to a hard wall) is qualitatively similar to that for the power-law confinement [compare Figs. 1(a) and 1(b)], the power-law confinement does not show the state with one chain (except for $\alpha=2$ and narrow range of $\eta$ ), and the fourchain state (between three and two chains) rapidly shrinks with increasing $\alpha$. The latter can be the reason that the non-SOT can hardly be detected in channels with power-law confinement in the limit of hard walls, and instead a usual SOT is observed (e.g., in dusty plasma [24]).

\section{Gaussian confinement}

An even softer transition between parabolic and hard-wall potential is presented in this section. Here we present a model where the confinement is presented by two Gaussians, symmetrically positioned with respect to the center of the channel. The shape of the confinement is controlled by the parameter $\gamma$, as shown in Eq. (5). For a specific value of $\gamma \approx 1$ it represents an approximation to the Bean-Livingston barrier [32,33] for vortices interacting with the boundary of a superconductor.

The phase diagram of a system of particles confined by $V_{C}(\gamma, y)$ is presented in Fig. 5 as a function of $\gamma$ and $\eta$. The best fit to a parabolic potential is provided by choosing $\gamma=0.34$. Then the evolution of the GS is guided by a non-SOT when density increases, as shown in the lower inset of Fig. 5 where the $y$ coordinate of the particles is plotted as a function of $\eta$.

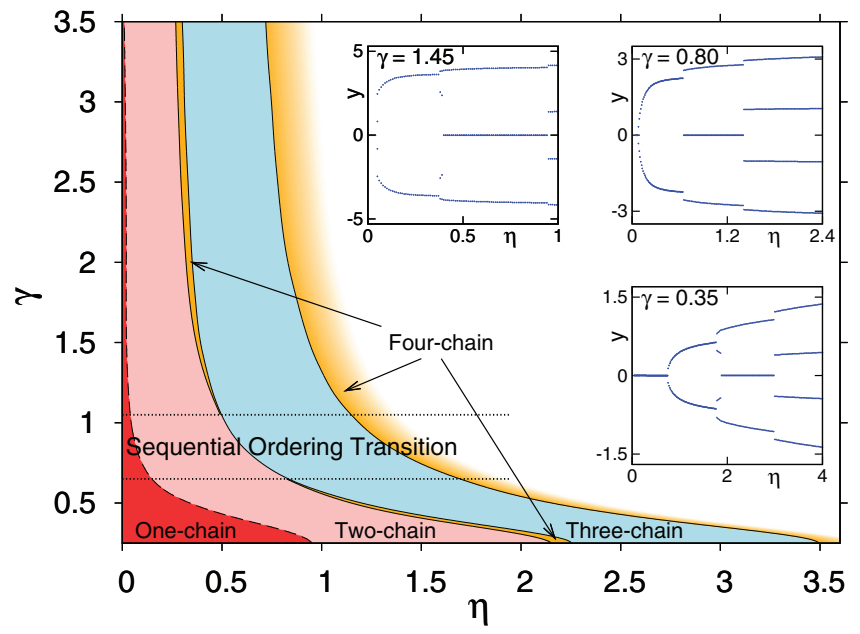

FIG. 5. (Color online) Phase diagram of the ground state for a system with confinement $V_{C}(\gamma, y)$, as a function of $\gamma$ and the linear density $\eta$. The solid and dashed lines represent first- and second-order transitions, respectively, while the region enclosed by the pointed lines indicates the sequential ordering transition. The insets show the $y$ position of the particles as a function of $\eta$ for different values of $\gamma$ as indicated in each figure.
As one can expect, in this case the GS undergoes a similar series of transitions as in the case of parabolic confinement.

However, as one can see from Fig. 5, the narrow region of the intermediate four-chain structure gradually shrinks with increasing $\gamma$ and around $\gamma=0.61$ this region disappears thus allowing a straightforward first-order transition from a twoto a three-chain configuration. Therefore, we observe a SOT in the system, as shown in the upper right-hand side inset of Fig. 5 where the transversal position of the particles is plotted as a function of the linear density.

On the other hand, this SOT is only observed as the GS for a certain range of the parameter $\gamma$, namely, from $\gamma=0.61$ to $\gamma=1.05$. For larger $\gamma$ values, the intermediate four-chain configuration is restored thus preventing the direct transition between two- and three-chain configurations. An important result is that this range includes the shape that fits the Bean-Livingston barrier for superconducting vortices. While we are not aware of any other explanations why the non-SOT has never been observed in superconducting slabs, our numerical result clearly indicates that non-SOT does not exist in a system of vortices confined by Bean-Livingston barriers in a superconducting stripe.

\section{CONCLUSIONS}

In this work, we studied the GS transitions of a system of particles interacting through a screened Yukawa potential and confined in a Q1D channel, where the structures found correspond to Wigner crystal configurations.

The effect of the confinement on the GS transitions, for increasing system density, is analyzed for different confinement profiles: the power-law $\left(\sim|y|^{\alpha}\right)$, exponential, and Gaussian potentials modulating the transversal profile of the channel through a shape parameter. Analytical expression for the energy of different $n$-chain configurations are calculated, and the GS is found by minimization of the energy with respect to the order parameter(s) of each analyzed structure.

As reference limiting cases, we defined a parabolic ("soft" confinement) and a hard-wall confinement, and the proposed potential profiles are able to transit continuously between these two limits. While asymptotically resembling each other, the different profiles evolve in a different manner for intermediate values of the shape parameters. This resulted in different sets of GS configurations which were analyzed in detail and summarized in phase diagrams "shape parameter versus density," for each considered confinement potential. Our choice of the model confinements was guided by those found in different physical systems, i.e., particles in a quasi-one-dimensional channel, when increasing the channel width. In particular, these correspond to charged particles in a parabolic trap which are realized, e.g., in experiments with dusty plasmas, colloids confined in narrow channels, or even vortices in superconducting stripes.

As follows from our analysis, due to the above similarity of the profiles for the limiting cases, all the systems display a similar behavior in the two limiting cases. Thus, the ground states of the systems with paraboliclike confinement profiles always evolve following a non-SOT. Similarly, all three systems allow a non-SOT in the hard-wall limit, although in the case of power-law confinement, as mentioned above, the 
one-chain configuration is missing. Simultaneously, we found that the non-SOT is present in all the systems for intermediate values of the shape parameter thus indicating that the non-SOT is extremely robust for a broad range of possible profiles and shape parameters.

At the same time, for the Gaussian confinement potential, a striking SOT for the GS was found to appear within a window of the shape parameter (i.e., $0.61<\gamma<1.05$ ). It is worth noting that this window includes the shape that describes the Bean-Livingston barrier for superconducting vortices. This result shows that the non-SOT does not exist in a system of vortices confined by Bean-Livingston barriers in a superconducting stripe and thus explains why the non-SOT, which was shown to be robust for different confinements, was never found for superconducting vortices.

Note that in many physical systems under real conditions, fluctuations may destroy the intermediate four-chain configuration which is probably the reason why the SOT (but not the non-SOT) has been observed in several experiments with colloids, dusty plasmas, and electrons in narrow channels. One indication that these thermal fluctuations are responsible for destroying the non-SOT for the Wigner crystal, was found numerically where it was shown that the non-SOT is present only for very low temperatures. In addition, it was recently found that a fluctuating number of particles in narrow short channels destroys the non-SOT while it is present in long narrow channels.

Thus our findings open the possibilities for using the confinement potential to manipulate the GS transition in the Q1D Wigner crystals and also can stimulate further studies in the field, both in theory and experiment.

\section{ACKNOWLEDGMENTS}

This work was supported by the Flemish Science Foundation (FWO-Vl) and the Odysseus and Methusalem programmes of the Flemish government. Computational resources were provided by HPC infrastructure of the University of Antwerp (CalcUA), a division of the Flemish Supercomputer Center (VSC).
[1] E. Wigner, Phys. Rev. 46, 1002 (1934).

[2] R. W. Hasse and V. V. Avilov, Phys. Rev. A 44, 4506 (1991).

[3] Y. G. Cornelissens, B. Partoens, and F. M. Peeters, Physica E 8, 314 (2000).

[4] V. A. Schweigert and F. M. Peeters, Phys. Rev. B 51, 7700 (1995).

[5] B. Partoens, V. A. Schweigert, and F. M. Peeters, Phys. Rev. Lett. 79, 3990 (1997).

[6] J. S. Meyer and K. A. Matveev, J. Phys.: Condens. Matter 21, 023203 (2009).

[7] H. J. Schulz, Phys. Rev. Lett. 71, 1864 (1993).

[8] V. V. Deshpande, M. Bockrath, L. I. Glazman, and A. Yacoby, Nature (London) 464, 209 (2010).

[9] G. Piacente, I. V. Schweigert, J. J. Betouras, and F. M. Peeters, Solid State Commun. 128, 57 (2003).

[10] G. Piacente, I. V. Schweigert, J. J. Betouras, and F. M. Peeters, Phys. Rev. B 69, 045324 (2004).

[11] G. Piacente and F. M. Peeters, Phys. Rev. B 72, 205208 (2005).

[12] G. Piacente, G. Q. Hai, and F. M. Peeters, Phys. Rev. B 81, 024108 (2010).

[13] J. E. Galván-Moya and F. M. Peeters, Phys. Rev. B 84, 134106 (2011).

[14] S. Fishman, G. De Chiara, T. Calarco, and G. Morigi, Phys. Rev. B 77, 064111 (2008).

[15] G. De Chiara, A. del Campo, G. Morigi, M. B. Plenio, and A. Retzker, New J. Phys. 12, 115003 (2010).

[16] H. Ikegami, H. Akimoto, and K. Kono, Phys. Rev. B 82, 201104 (2010).

[17] J. S. Meyer, K. A. Matveev, and A. I. Larkin, Phys. Rev. Lett. 98, 126404 (2007).

[18] T. Meng, M. Dixit, M. Garst, and J. S. Meyer, Phys. Rev. B 83, 125323 (2011).

[19] E. Shimshoni, G. Morigi, and S. Fishman, Phys. Rev. A 83, 032308 (2011).
[20] A. Bermudez and M. B. Plenio, Phys. Rev. Lett. 109, 010501 (2012).

[21] C. Cormick and G. Morigi, Phys. Rev. A 87, 013829 (2013).

[22] M. Köppl, P. Henseler, A. Erbe, P. Nielaba, and P. Leiderer, Phys. Rev. Lett. 97, 208302 (2006).

[23] A. D. Klironomos and J. S. Meyer, Phys. Rev. B 84, 024117 (2011).

[24] T. E. Sheridan and K. D. Wells, Phys. Rev. E 81, 016404 (2010).

[25] J. Guimpel, L. Civale, F. de la Cruz, J. M. Murduck, and I. K. Schuller, Phys. Rev. B 38, 2342 (1988).

[26] S. H. Brongersma, E. Verweij, N. J. Koeman, D. G. de Groot, R. Griessen, and B. I. Ivlev, Phys. Rev. Lett. 71, 2319 (1993).

[27] G. Carneiro, Phys. Rev. B 57, 6077 (1998).

[28] E. Sardella, M. M. Doria, and P. R. S. Netto, Phys. Rev. B 60, 13158 (1999)

[29] J. Barba-Ortega, A. Becerra, and J. A. Aguiar, Physica C 470, 225 (2010).

[30] E. Bronson, M. P. Gelfand, and S. B. Field, Phys. Rev. B 73, 144501 (2006).

[31] W. P. Ferreira, J. C. N. Carvalho, P. W. S. Oliveira, G. A. Farias, and F. M. Peeters, Phys. Rev. B 77, 014112 (2008).

[32] M. Tinkham, Introduction to Superconductivity, 2nd ed. (McGraw-Hill, New York, 1966).

[33] C. P. Bean and J. D. Livingston, Phys. Rev. Lett. 12, 14 (1964).

[34] H. Ikegami, H. Akimoto, D. G. Rees, and K. Kono, Phys. Rev. Lett. 109, 236802 (2012).

[35] A. A. Vasylenko and V. R. Misko, Biophys. Rev. Lett. (2014), doi:10.1142/S1793048014400037.

[36] C. Reichhardt and C. J. Olson Reichhardt, Phys. Rev. E 85, 051401 (2012).

[37] B. Liu, K. Avinash, and J. Goree, Phys. Rev. Lett. 91, 255003 (2003). 
[38] G. Coupier, M. Saint Jean, and C. Guthmann, Phys. Rev. E 73, 031112 (2006).

[39] P. Galatola, G. Coupier, M. Saint Jean, J. B. Fournier, and C. Guthmann, Eur. Phys. J. B 50, 549 (2006).

[40] J. B. Delfau, C. Coste, C. Even, and M. Saint Jean, Phys. Rev. E 82, 031201 (2010).
[41] C. Coste, J. B. Delfau, C. Even, and M. Saint Jean, Phys. Rev. E 81, 051201 (2010).

[42] C.-H. Sow, K. Harada, A. Tonomura, G. Crabtree, and D. G. Grier, Phys. Rev. Lett. 80, 2693 (1998).

[43] D. V. Tkachenko, T. E. Sheridan, and V. R. Misko, Phys. Plasmas 18, 103709 (2011) 